\title{
MiR-126-3p inhibits apoptosis and promotes proliferation by targeting phosphatidylinositol 3-kinase regulatory subunit 2 in porcine ovarian granulosa cells
}

\author{
Xiaofeng Zhou', Yingting He ${ }^{1}$, Yao Jiang ${ }^{1}$, Bo He ${ }^{1}$, Xi Deng ${ }^{1}$, Zhe Zhang ${ }^{1}$, Xiaolong Yuan ${ }^{1, *}$, and Jiaqi $\mathrm{Li}^{1, *}$
}

\footnotetext{
* Corresponding Authors:

Xiaolong Yuan

Tel: +86-0-20-85282019, Fax: +86-0-20-85282019,

E-mail: yxl@scau.edu.cn

Jiaqi Li

Tel: +86-0-20-85283519, Fax: +86-0-20-85283519

E-mail: jqli@scau.edu.cn
}

${ }^{1}$ Guangdong Provincial Key Lab of Agro-Animal Genomics and Molecular Breeding, National

Engineering Research Centre for Breeding Swine Industry, College of Animal Science, South China Agricultural University, Guangzhou, Guangdong 510642, China

ORCID

Xiaofeng Zhou

https://orcid.org/0000-0002-7847-4383 Yingting $\mathrm{He}$

https://orcid.org/0000-0003-3004-1308 Yao Jiang

https://orcid.org/0000-0003-3065-2467

Bo He

https://orcid.org/0000-0002-5866-2192

Xi Deng

https://orcid.org/0000-0003-2124-8534

Zhe Zhang

https://orcid.org/0000-0001-7338-7718

Xiaolong Yuan

https://orcid.org/0000-0002-3743-0130

Jiaqi Li

https://orcid.org/0000-0002-8308-9850

Submitted Apr 7, 2019; Revised Jun 15, 2019; Accepted Aug 14, 2019
Objective: Numerous studies have indicated that the apoptosis and proliferation of granulosa cells (GCs) are closely related to the normal growth and development of follicles and ovaries. Previous evidence has suggested that miR-126-3p might get involved in the apoptosis and proliferation of GCs, and phosphatidylinositol 3-kinase regulatory subunit 2 (PIK3R2) gene has been predicted as one target of miR-126-3p. However, the molecular regulation of miR126-3p on PIK3R2 and the effects of PIK3R2 on porcine GCs apoptosis and proliferation remain virtually unexplored.

Methods: In this study, using porcine GCs as a cellular model, luciferase report assay, mutation and deletion were applied to verify the targeting relationship between miR-126-3p and PIK3R2. Annexin-V/PI staining and 5-ethynyl-2'-deoxyuridine assay were applied to explore the effect of PIK3R2 on GCs apoptosis and proliferation, respectively. Real-time quantitative polymerase chain reaction and Western Blot were applied to explore the regulation of miR126-3p on PIK3R2 expression.

Results: We found that miR-126-3p targeted at PIK3R2 and inhibited its mRNA and protein expression. Knockdown of PIK3R2 significantly inhibited the apoptosis and promoted the proliferation of porcine GCs, and significantly down-regulated the mRNA expression of several key genes of PI3K pathway such as insulin-like growth factor 1 receptor (IGF1R), insulin receptor (INSR), pyruvate dehydrogenase kinase 1 (PDK1), and serine/threonine kinase 1 (AKT1).

Conclusion: MiR-126-3p might target and inhibit the mRNA and protein expressions of PIK3R2, thereby inhibiting GC apoptosis and promoting GC proliferation by down-regulating several key genes of the PI3K pathway, IGF1R, INSR, PDK1, and AKT1. These findings would provide great insight into further exploring the molecular regulation of miR-126-3p and PIK3R2 on the functions of GCs during the folliculogenesis in female mammals.

Keywords: Ovarian Granulosa Cells; miR-126-3p; Phosphatidylinositol 3-Kinase Regulatory Subunit 2 (PIK3R2); Cell Apoptosis, Cell Proliferation

\section{INTRODUCTION}

Granulosa cells (GCs) have been shown to play an important role in the dynamic process of folliculogenesis, including follicular initiation [1], dominance [2], ovulation [3], luteinization [4] and atresia [5,6] by regulating proliferation, cell cycle, apoptosis, and synthesis of steroid hormones [7] in female mammals. These processes are important to the maturation of oocyte and propagation of species. Previous studies have reported that the estrogen secreted by GCs is reduced in estrogen receptor 2 mutant mice, resulting in impaired follicle maturation and failure of ovulation [8-10], and moreover, the high apoptosis ratio of GCs could impair folliculogenesis and give rise to the increase follicular atresia $[11,12]$. These 
observations show that GCs are closely related to the development of follicles and ovaries.

The microRNA is a class of single-stranded non-coding RNA consisting of about 22 nucleotides in length that regulates the expression of the target gene at the post-transcriptional level [13]. Studies have shown that a variety of miRNAs are involved in the development of ovarian follicles, corpus luteum formation, degeneration as well as follicular atresia $[14,15]$. For example, in mouse GCs, miR-224 targets SMAD family member 4 (Smad4) to promote the proliferation of GCs and the expression of aromatase cytochrome P450, family 19, subfamily a, polypetide1 (Cyp19a1) through the transforming growth factor, beta 1 (TGF $\beta 1$ )/Smads pathway, thereby modulating the secretion of estradiol [16]. In pigs, miR-26b was differentially expressed between normal and atresia follicles, and has been proved to promote GCs apoptosis by targeting DNA damage related genes ATM serine/threonine kinase $(A T M)[17,18]$. In bovine, miR-378 may suppress the apoptosis of luteal cells by targeting interferon gamma receptor 1 gene [19]. However, the molecular mechanisms of how miRNAs regulate GCs proliferation and apoptosis remain unclear in mammals.

A recent study has reported that miR-126-5p is significantly decreased in GCs of polycystic ovarian syndrome (PCOS) patients, compared to healthy women, and may be involved in GC apoptosis [20]. In our previous study, we have proved that miR-126-3p inhibits apoptosis and promotes proliferation of porcine ovarian GCs [21]. These results suggested that miR-126-3p might exhibit an essential role in apoptosis and proliferation of porcine GCs, and consequently give rise to dynamic impact on the development of follicles. In this study, we found that phosphatidylinositol 3-kinase regulatory subunit 2 (PIK3R2) was a potential target of miR-126-3p by using bioinformatic algorithms. PIK3R2 is a member of phosphatidylinositol kinase (PI3K) 3-family gene [22]. It is reported that PIK3R2 can encode $\mathrm{p} 85 \beta$, an enzyme that generates 3-polyphosphoinositides at the plasma membrane, to inhibit phosphorylation of Akt and resulting in suppressing PI3K/ AKT proliferation-survival signaling pathway $[23,24]$. In rheumatoid arthritis synovial fibro-blasts cell, PIK3R2 inhibited the proliferation and promoted the apoptosis by regulating PI3K/AKT pathway [25]. However, the function of PIK3R2 in porcine GCs has not been reported. Therefore, we hypothesized that miR-126-3p might target PIK3R2 to regulate proliferation and apoptosis of porcine ovarian GCs. In this study, we aimed to investigate whether miR-126-3p targeted and repressed the expression of PIK3R2, and consequently regulated the proliferation and apoptosis of porcine ovarian GCs.

\section{MATERIALS AND METHODS}

Ethics approval

All experiments conducted in this study strictly followed the guidelines of the Animal Care and Use Committee of South China Agricultural University Guangzhou, China (approval number: SCAU\#2013-10).

\section{Ovarian granulosa cell culture and transient transfection}

The ovaries of pre-puberty sows were collected from a local slaughterhouse and transported to the laboratory using phosphate buffered saline (PBS) containing penicillin (100 IU/mL) and streptomycin $(100 \mu \mathrm{g} / \mathrm{mL})$ (Invitrogen, Shanghai, China). Subsequently, the GC was aspirated by inserting a syringe into a 3 to $5 \mathrm{~mm}$ follicle, and the separated GCs were washed twice with PBS. The cells were then seeded into culture flasks containing $10 \%$ fetal bovine serum (Hyclone, Logan, UT, USA) in Dulbecco's modified eagle medium (DMEM) (Hyclone, USA) and $100 \mathrm{IU} / \mathrm{mL}$ penicillin, $100 \mu \mathrm{g} / \mathrm{mL}$ streptomycin, and finally incubated at $37^{\circ} \mathrm{C}$ under $5 \% \mathrm{CO}_{2}$. After $24 \mathrm{~h}$ of culturing GCs, the cell confluence reached $70 \%$ to $90 \%$ for transfection (Figure 1A). The GCs were transfected with miR-126-3p inhibitor, inhibitor negative control (NC), siRNAPIK3R2, or siRNA-NC for $48 \mathrm{~h}$ using Lipofectamine 3000 Reagent (Invitrogen, China).

\section{Real-time quantitative polymerase chain reaction analysis}

TRIzol reagent (TaKaRa, Tokyo, Japan) was used to extract total RNA from the sample, and then the RevertAid First Strand cDNA Synthesis Kit (Thermo Scientific, Waltham, MA, USA) was used to reverse-transcribe the mRNAs. Maxima SYBR Green qRT-PCR Master Mix (2x) (Thermo Scientific, USA) was used to quantify the relative expression levels of mRNAs in the LightCycler real-time polymerase chain reaction (PCR) system. Using the expression level of glyceraldehyde 3-phosphate dehydrogenase (GAPDH) as endogenous control, the relative expression level of PIK3R2 was calculated with the $2-\Delta \Delta$ ct method. The Primer sequences are listed in Table 1.

\section{Cell proliferation and apoptosis assay}

The Cell-LightTM 5-ethynyl-2'-deoxyuridine (EdU) Apollo 567 In Vitro Kit (RiboBio Co., Ltd., Guangzhou, China) was used to analyze cell proliferation. Briefly, GCs were cultured in 48-well plates and transfected with plasmid for $36 \mathrm{~h}$. The GCs were incubated at room temperature with $50 \mu \mathrm{M}$ EdU for $2 \mathrm{~h}$, washed twice with PBS, and then incubated with $80 \%$ acetone for $30 \mathrm{~min}$. After GCs were washed twice with PBS, $0.5 \%$ Triton X-100 was added for $10 \mathrm{~min}, 1 \times$ Apollo was incubated in darkness for $30 \mathrm{~min}$, and Hoechst was incubated for $30 \mathrm{~min}$. Finally, three fields were randomly selected from each well and GCs were counted under an inverted fluores- 
A

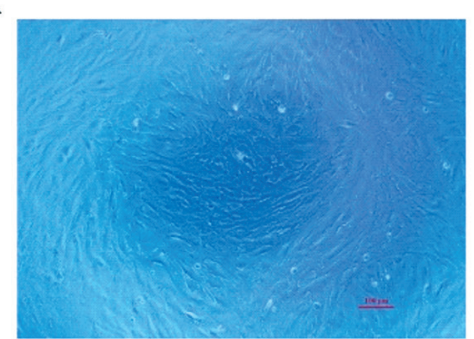

D

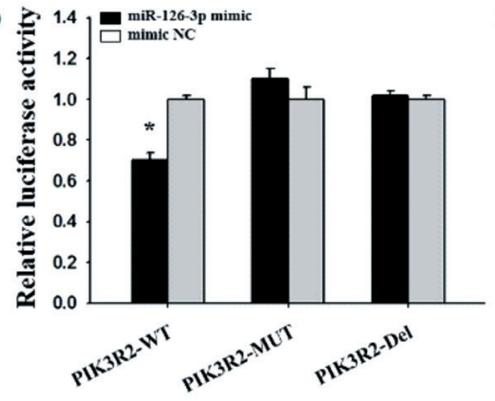

B
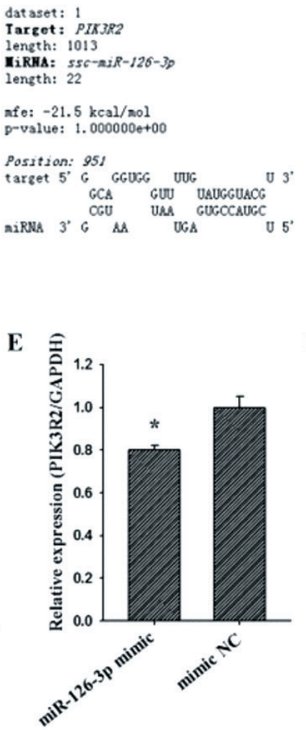

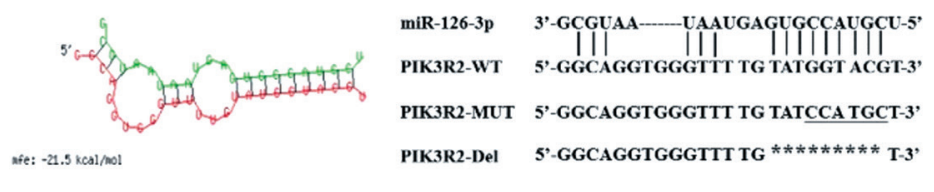

G

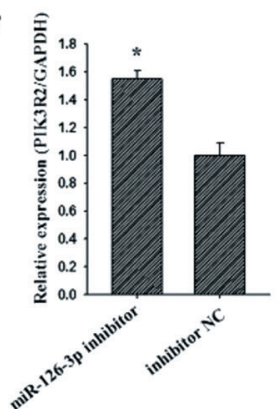

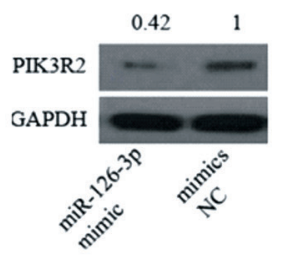

H

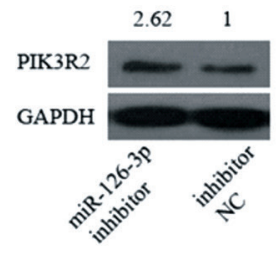

Figure 1. PIK3R2 is a target gene of miR-126-3p. The cultured porcine GCs for $24 \mathrm{~h}(\mathrm{~A})$. A miR-126-3p binding site within the PIK3R2 3'-UTR is predicted by RNAhybrid (B). Wild-type sequence (PIK3R2-WT), mutated sequence (PIK3R2-MUT), and deleted sequence (PIK3R2-Del) of the miR-126-3p binding site (C). Relative luciferase activities of PIK3R2-WT, PIK3R2-MUT, and PIK3R2-Del after transfection with the miR-126-3p mimics or mimic NC in GCs (D). Relative mRNA expression of PIK3R2 after transfection with the miR-126-3p mimics (E) and inhibitors ( $F$ ) in GCs. Relative protein expression of PIK3R2 after transfection with the miR-126-3p mimics (G) and inhibitors (H) in GCs. The scale bar of the micrograph is $100 \mu \mathrm{m}$. PIK3R2, phosphatidylinositol 3-kinase regulatory subunit 2; GCs, granulosa cells; GAPDH, glyceraldehyde 3-phosphate dehydrogenase. * indicates $p<0.05$. Mimic NC is the negative control of miR-126-3p mimic and inhibitor NC is the negative control of miR-126-3p inhibitor. The relative protein expression level of PIK3R2 was normalized by GAPDH values.

cence microscope.

The Annexin V-FITC Apoptosis Detection Kit (BioVision, Milpitas, CA, USA) was used to analyze cell apoptosis. Briefly, GCs were cultured in 6-well and transfected with plasmids for $48 \mathrm{~h}$. The collected cells were centrifuged at 1,000 rpm for 5 min, supernatant discarded, and washed twice with PBS. Then, $500 \mu \mathrm{L}$ of $1 \mathrm{X}$ Annexin V buffer was added to gently resuspend the cells, and $5 \mu \mathrm{L}$ of Annexin V-FITC and $5 \mu \mathrm{L}$

Table 1. Primers used in the present study

\begin{tabular}{llc}
\hline Name & \multicolumn{1}{c}{ Sequence } & Product (bp) \\
\hline qRT-PIK3R2-F & GACTGTGGGATTGAGACG & 145 \\
qRT-PIK3R2-R & ACCCGAGTAAGAATGTGC & \\
qRT-IGF1R-F & AAAGGATGAAGTCTGGCTCC & 209 \\
qRT-IGF1R-R & TCAGCCTTGGAGATGAGCAG & \\
qRT-INSR-F & CACTGGCTATCGCATTGAGC & 173 \\
qRT-INSR-R & CCTGCCACATCAAGTGAACG & \\
qRT-PDK1-F & ACATGTACTCCACTGCACCC & 91 \\
qRT-PDK1-R & AGGCGTGATATGGGCAATCC & \\
qRT-AKT1-F & AGAAGCTCTTCGAGCTCATCCTCA & 148 \\
qRT-AKT1-R & TGCATGATCTCCTTGGCATCCTCA & \\
qRT-GADPH-F & GGACTCATGACCACGGTCCAT & 220 \\
qRT-GADPH-R & TCAGATCCACAACCGACACGT & \\
SiRNA-PIK3R2-1 & GGAGAAGUUACUUCAGGAA & \\
SiRNA-PIK3R2-2 & GGAACAACAAGCUGAUCAA & \\
SiRNA-PIK3R2-3 & GGUAUGUAGGCAAGAUCAA & \\
\hline
\end{tabular}

of propidium iodide staining solution were added and mixed. Finally, flow cytometry was performed after incubation for 15 $\mathrm{min}$ at room temperature in darkness. For results, the figure has four quadrants, the lower right quadrant is annexinpositive/PI-negative early apoptotic cells, the upper right quadrant is annexin-positive/PI-positive late apoptotic cells, the lower left quadrant is living cell, and the upper left quadrant is mechanical injury cells. In this study, the apoptotic ratio of GCs is the sum of early and late apoptosis.

Vector construction and dual-luciferase reporter assay The region of PIK3R2 gene 3'-UTR that contains a potential binding site of miR-126-3p was cloned and ligated it to the pmirGLO dual luciferase miRNA target expression vector (Promega, Madison, WI, USA). We constructed three recombinant vectors, the recombinant vector containing the wildtype miR-126-3p binding site sequence was named as PIK3R2wild-type (WT), the mutant vector containing part of the miR-126-3p binding site sequence was named as PIK3R2mutant, and the recombinant vector deleting part of the miR126-3p binding site sequence was named as PIK3R2-deleted (Del). The GCs were cultured in 24-well plates and transfected with the successfully constructed vector for 48 hours, and finally the Dual-Glo Luciferase Assay Kit (Promega, USA) was used to test the relative luciferase activity. 


\section{Western blot analysis}

Total protein was isolated from the GC samples and the BCA Protein Assay Kit (Vigorous Bio-technology Beijing Co., Ltd., Beijing, China) was used to quantitate the amount of protein. Then, after denaturation by boiling with $5 \times$ protein sodium dodecyl sulfate-polyacrylamide gel electrophoresis (SDSPAGE) loading buffer for $10 \mathrm{~min}$, the protein samples were separated by SDS-PAGE and transferred onto nitrocellulose membrane. The membranes were incubated with anti-hamartin primary antibody $(1: 1,000$; Biorbyt, San Francisco, CA, USA). Following incubation with the secondary antibody for 1 hour at room temperature, the ECL-PLUS kit (Amersham Biosciences, Piscataway, NJ, USA) was used to visualize antibody-bound protein bands. Among them, an anti-GAPDH antibody (1:3,000; Sigma, St. Louis, MO, USA) was used as an internal control. Finally, ImageJ software was used to calculate the gray value of the band and the relative protein expression level of PIK3R2 was normalized by GAPDH value.

\section{Data analysis}

All statistical analyses were performed with $\mathrm{R}$ software and data were presented as means \pm standard deviation from at least three independent experiments. The significance of differences in means between two groups was analyzed by using Student's t-test (two-tailed). ${ }^{*}$ indicates $\mathrm{p}<0.05 ;{ }^{*}$ indicates $\mathrm{p}<0.01$.

\section{RESULTS}

miR-126-3p targets at PIK3R2 and inhibits its mRNA and protein expression

We found PIK3R2 was a target gene of miR-126-3p by using three bioinformatics algorithms, TargetScan, miRanda, and RNAhybrid (Figure 1B). To further confirm whether miR-126$3 \mathrm{p}$ was targeting PIK3R2, we co-transfected the constructed recombinant vectors PIK3R2-WT, PIK3R2-mutant (MUT) and PIK3R2-Del (Figure 1C) into GCs (Figure 1A) with miR126-3p mimic or mimic NC, respectively. As shown in Figure $1 \mathrm{D}$, we found that the luciferase activity of the miR-126-3p mimics in PIK3R2-WT was significantly lower than mimic $\mathrm{NC}(\mathrm{p}<0.05)$, but the luciferase activity of the miR-126-3p mimics in PIK3R2-MUT and PIK3R2-Del showed no significant difference with mimic NC in GCs (Figure 1D). These results indicated that miR-126-3p inhibited the luciferase activity of the reporter gene by binding to the $3^{\prime} \mathrm{UTR}$ of PIK3R2.

To further explore the effects of miR-126-3p on the expression of PIK3R2, miR-126-3p mimic or miR-126-3p inhibitor was transfected into porcine GCs. Compared with mimic NC, miR-126-3p mimic significantly down-regulated the mRNA (Figure 1E, $\mathrm{p}<0.05$ ) and protein (Figure $1 \mathrm{G}$ ) level of PIK3R2. Compared with inhibitor NC, miR-126-3p inhibitor significantly up-regulated the mRNA (Figure $1 \mathrm{~F}, \mathrm{p}<0.05$ ) and protein (Figure $1 \mathrm{H}$ ) levels of PIK3R2. These observations indicated that miR-126-3p may target PIK3R2 3'UTR and repress its mRNA and protein expression level in porcine GCs.

\section{Knockdown PIK3R2 inhibits GCs apoptosis and promotes GCs proliferation}

To investigate the cellular function of PIK3R2 on GCs apoptosis and proliferation, three PIK3R2-specific small interfering RNAs (siRNA) (siRNA-PIK3R2-1, siRNA-PIK3R2-2, siRNAPIK3R2-3) and negative control (siRNA-NC) were transfected into GCs (Figure 2A). As shown in Figure 2A, siRNA-PIK3R2-1 exhibited the best inhibition efficiency, and thus siRNAPIK3R2-1 was selected for knockdown PIK3R2 in GCs. As shown in Figure 2, the GC apoptosis rate of the siRNA-PIK3R2 group was significantly lower than siRNA-NC group (Figure $2 \mathrm{~B}, \mathrm{p}<0.01$ ), and the GC proliferation rate of the siRNAPIK3R2 group was significantly higher than siRNA-NC group (Figure $2 \mathrm{C}, \mathrm{p}<0.01$ ). Furthermore, several key genes of the PI3K pathway, insulin-like growth factor 1 receptor (IGF1R) [26], insulin receptor (INSR) [27], pyruvate dehydrogenase kinase 1 (PDK1) [28] and serine/threonine kinase 1 (AKT1) [29] were selected and detected to characterize biological functions of PIK3R2. We found that the mRNA expressions of IGF1R (Figure 2D, $\mathrm{p}<0.01$ ), INSR (Figure 2D, $\mathrm{p}<0.01$ ), $P D K 1$ (Figure 2D, $\mathrm{p}<0.01$ ), and $A K T 1$ (Figure 2D, $\mathrm{p}<0.05)$ in siRNA-PIK3R2 group were all significantly lower than that in siRNA-NC group. These observations demonstrated that knockdown PIK3R2 may inhibit GC apoptosis and promote GC proliferation by disturbing the PI3K pathway.

miR-126-3p regulates granulosa cells apoptosis and proliferation by targeting PIK3R2

To further determine whether miR-126-3p inhibited GCs apoptosis and promoted GCs proliferation by targeting PIK3R2, miR-126-3p inhibitor, inhibitor NC, siRNA-PIK3R2, and siRNA-NC were co-transfected into porcine GCs. For the apoptosis rate of porcine GCs (Figure 3A), group 1 (miR126-3p inhibitor + siRNA-PIK3R2) wasn't notably different from group 4 (inhibitor $\mathrm{NC}+$ siRNA-NC) $(\mathrm{p}>0.05)$, group 2 (inhibitor NC + siRNA-PIK3R2) was significantly lower than group $4(\mathrm{p}<0.05)$, and group 3 (miR-126-3p inhibitor + siRNA-NC) was significantly higher than group $4(\mathrm{p}<0.05)$. These findings suggest that knockdown miR-126-3p could reverse the siRNA-PIK3R2-mediated inhibition of GC apoptosis, indicating that miR-126-3p may target PIK3R2 to inhibit GCs apoptosis. For the proliferation rate of porcine GCs (Figure 3B), group 1 wasn't notably different from group 4 ( $p>0.05)$, group 2 was significantly higher than group 4 $(\mathrm{p}<0.05)$, and group 3 was significantly lower than group 4 $(\mathrm{p}<0.05)$. These findings suggest that knockdown miR-126$3 p$ could reverse the siRNA-PIK3R2-mediated promotion 

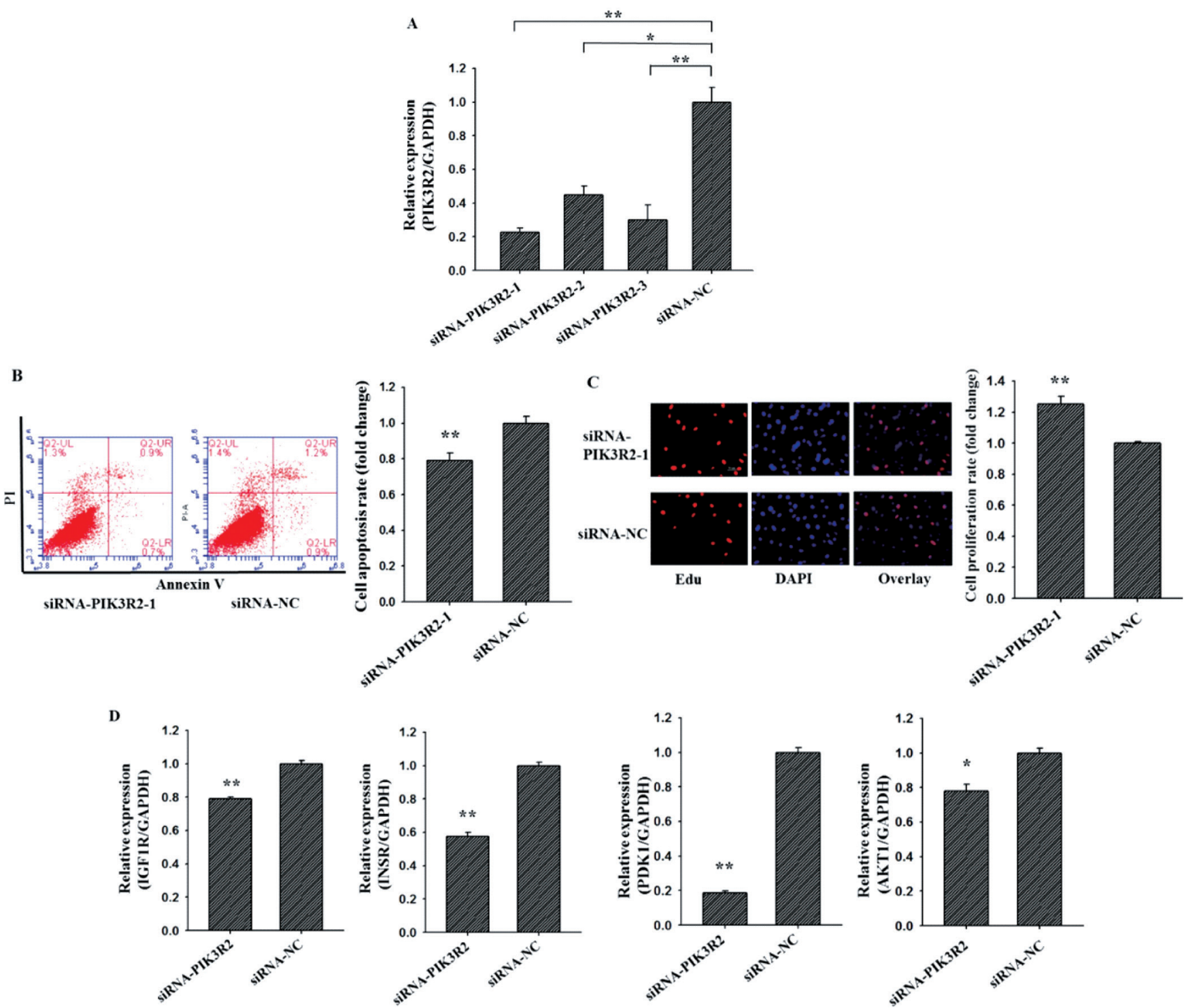

Figure 2. Knockdown PIK3R2 might inhibit GCS apoptosis and promote GCs proliferation by disturbing PI3K pathway. SiRNA-PIK3R2-1 exhibited the best inhibition efficiency of PIK3R2 by using quantitative RT-PCR (A). Knockdown PIK3R2 could inhibit GCs apoptosis by using Annexin-V/PI staining and flow cytometry analysis (B). Knockdown PIK3R2 could promote GCs proliferation by using EdU assays (C). Relative mRNA expression of several key genes of PI3K pathway, IGF1R, INSR, PDK1, and $A K T 1$, after transfection with the PIK3R2 inhibitors (D). PIK3R2, phosphatidylinositol 3-kinase regulatory subunit 2; GCs, granulosa cells; RT-PCR, real-time polymerase chain reaction; IGF1R, insulin-like growth factor 1 receptor; INSR, insulin receptor; PDK1, pyruvate dehydrogenase kinase 1; AKT1, serine/threonine kinase 1. * indicates $p<0.05$ and ${ }^{* *}$ indicates $p<0.01$. Mimic NC is the negative control of miR-126-3p mimic and inhibitor NC is the negative control of miR-126-3p inhibitor. Compared with control, the fold change of GCs proliferation or apoptosis rate is presented in Figure $2 \mathrm{~B}$ and $2 \mathrm{C}$.

of GC proliferation, indicating that miR-126-3p may target at PIK3R2 to promote GCs proliferation. The above results showed that miR-126-3p may inhibit GCs apoptosis and promote GCs proliferation by targeting PIK3R2.

\section{DISCUSSION}

Previous evidence has indicated that miR-126-3p plays important roles in the process of folliculogenesis, oogenesis, and steroidogenesis in different species. In bovine corpus luteum, miR-126-3p is an important regulator of talin 2 and partici- pates in luteal development during the estrous cycle [30]. In mouse, miR-126-3p can specifically inhibit progesterone receptor expression and $\beta$-casein secretion, then changes the viability of mammary epithelial cells and participates in mammary gland development [31]. In human primary ovarian GCs, miR-126-3p influences the mRNA level of apoptosis markers and suppresses progesterone, testosterone and estradiol secretion [32,33].

In our previous study, we have proved that miR-126-3p inhibits apoptosis and promotes proliferation of porcine ovarian GCs. In present study, the bioinformatics and luciferase 
A
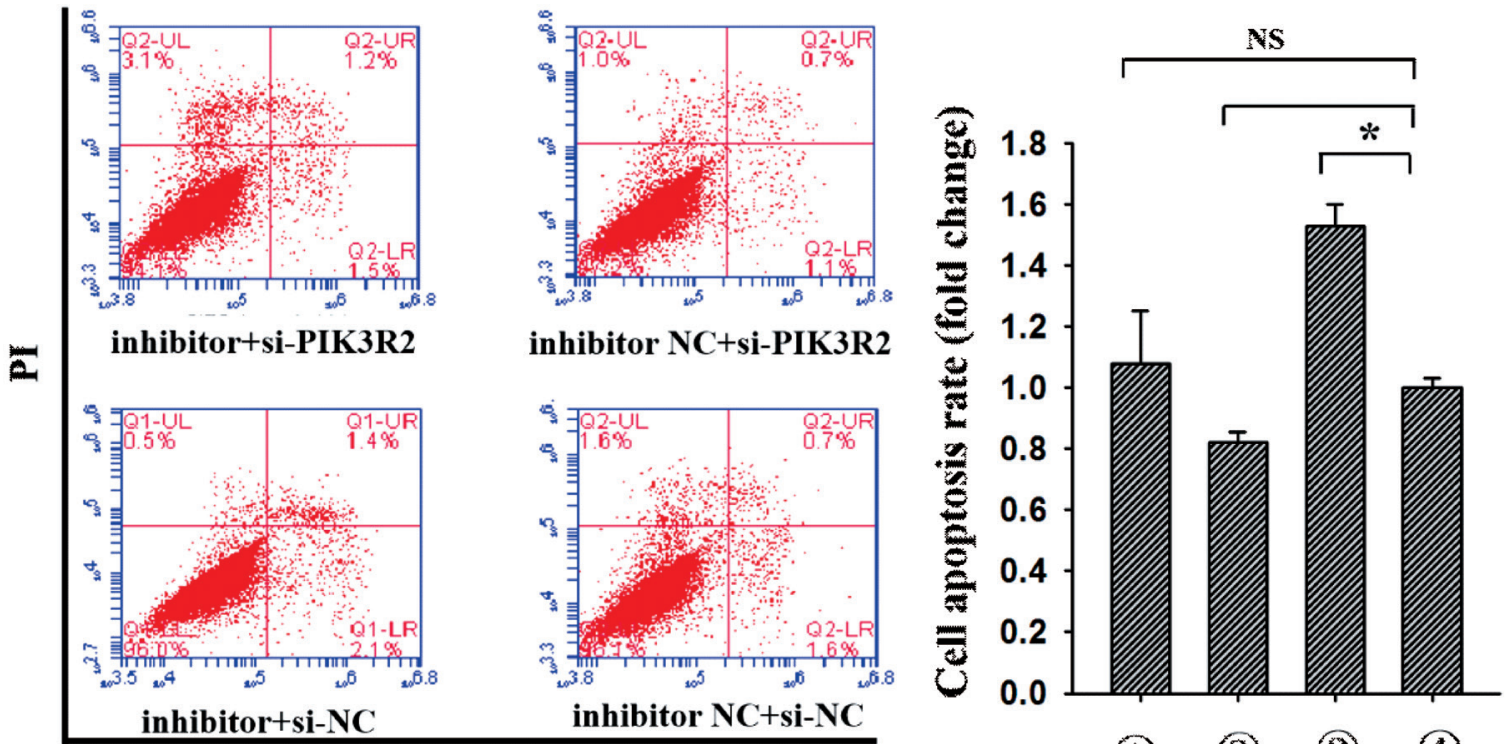

Annexin $\mathrm{V}$

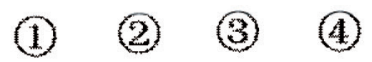

$\begin{array}{lllll}\text { miR-126-3p inhibitor } & + & - & + & - \\ \text { inhibitor NC } & - & + & - & + \\ \text { siRNA-PIK3R2 } & + & + & - & - \\ \text { siRNA-NC } & - & - & + & +\end{array}$

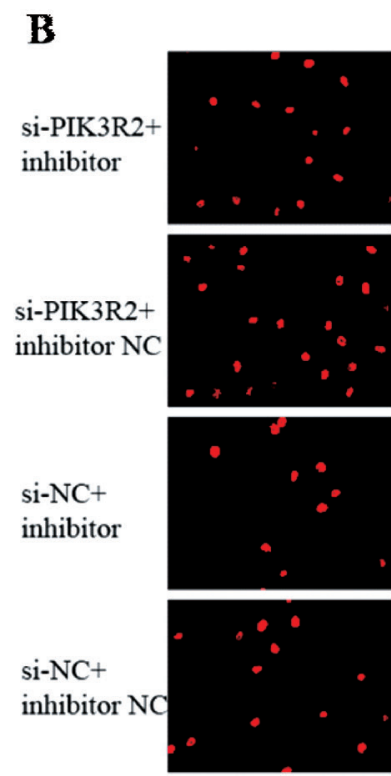

Edu
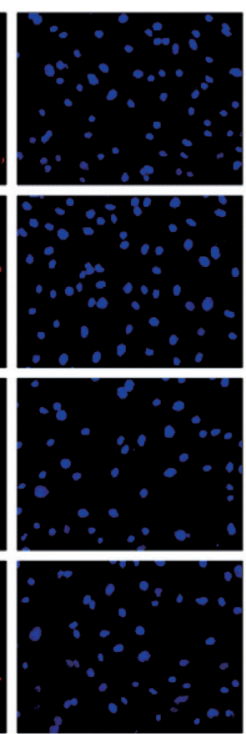

DAPI
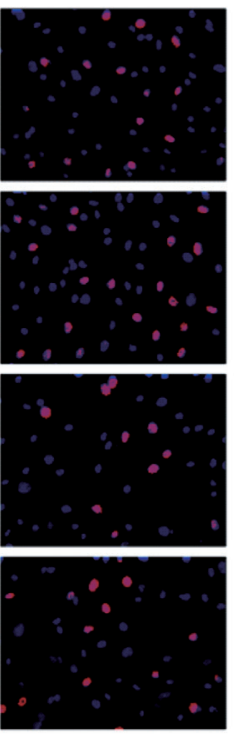

Overlay
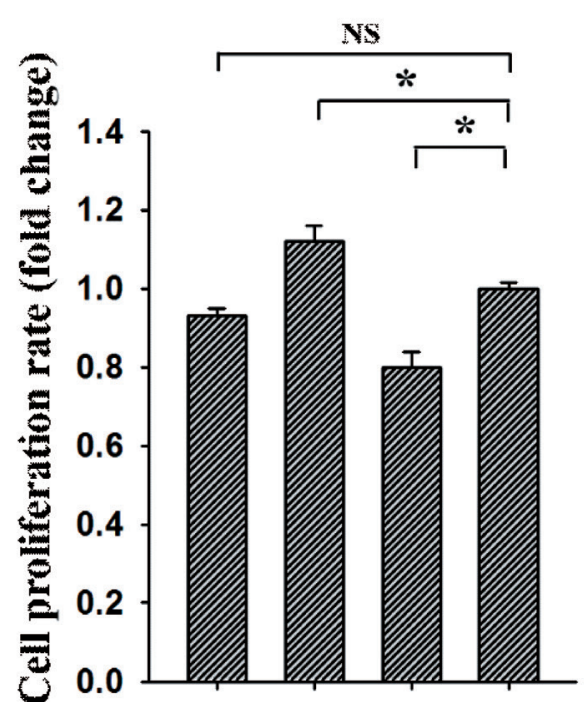

(1) (2)

(3)
(4)

$\begin{array}{lllll}\text { miR-126-3p inhibitor } & + & - & + & - \\ \text { inhibitor NC } & - & + & - & + \\ \text { siRNA-PIK3R2 } & + & + & - & - \\ \text { siRNA-NC } & - & - & + & +\end{array}$

Figure 3. miR-126-3p inhibits GCs apoptosis and promotes GCs proliferation by targeting PIK3R2. Annexin-V/PI staining and flow cytometry analysis showed that miR126-3p inhibited GCs apoptosis by targeting PIK3R2 (A). EdU assays indicated that miR-126-3p promoted GCs proliferation by targeting PIK3R2 (B). PIK3R2, phosphatidylinositol 3-kinase regulatory subunit 2; GCs, granulosa cells. * indicates $p<0.05$ and ** indicates $p<0.01$. Mimic NC is the negative control of miR-126-3p mimic and inhibitor NC is the negative control of miR-126-3p inhibitor. Compared with control, the fold change of GCs proliferation or apoptosis rate is presented in the Figure 3. 


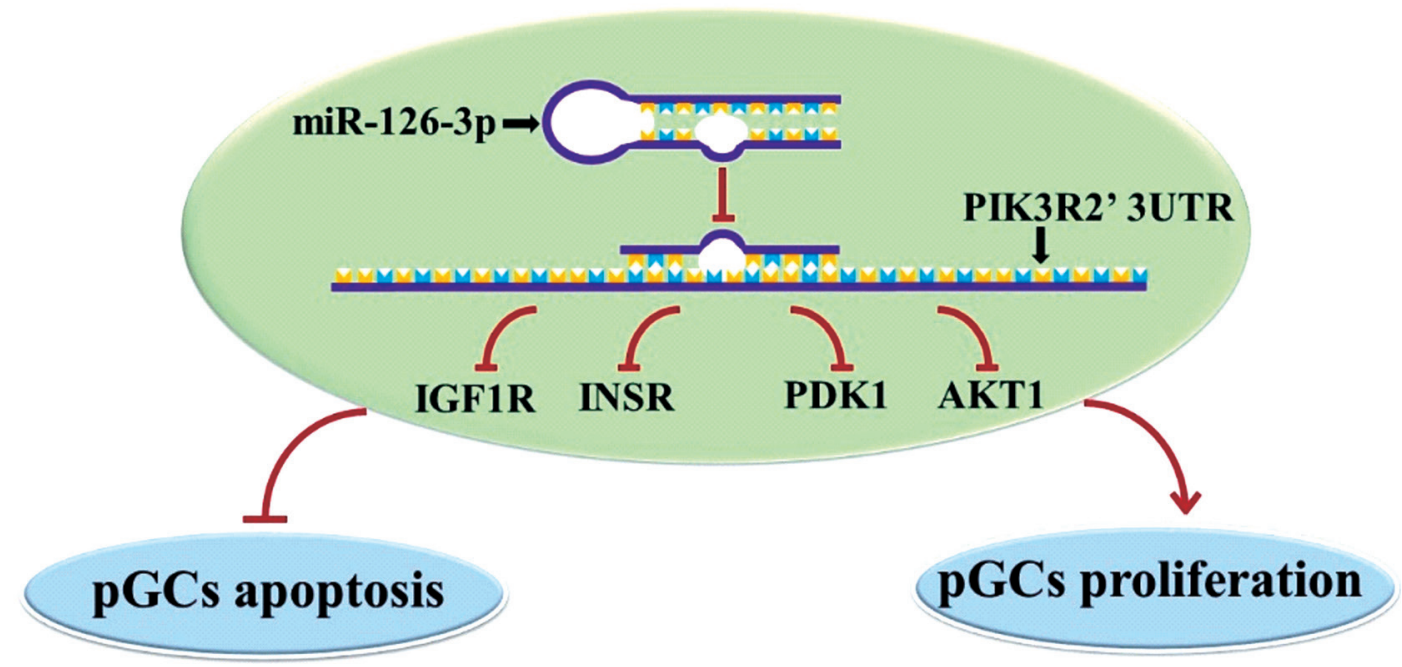

Figure 4. The potential regulatory modeling of miR126-3p and PIK3R2 in porcine GCs (pGCs). MiR-126-3p may target and inhibit the mRNA and protein expression of PIK3R2, thereby inhibiting several genes of PIK pathway, IGFIR, INSR, PDK1, and AKT1, which ultimately inhibit GCs apoptosis and promote GCs proliferation. PIK3R2, phosphatidylinositol 3-kinase regulatory subunit 2; GCs, granulosa cells; IGF1R, insulin-like growth factor 1 receptor; INSR, insulin receptor; PDK1, pyruvate dehydrogenase kinase 1; AKT1, serine/threonine kinase 1.

activity assay showed that miR-126-3p was directly targeted PIK3R2 3'UTR. Moreover, miR-126-3p can negatively regulate the expression of $P I K 3 R 2$ at post-transcriptional and translational levels (Figure 1). siRNA-mediated PIK3R2 knockdown could inhibit GC proliferation (Figure $3 \mathrm{~A}$ ) and promote GC apoptosis (Figure 3B), while miR-126-3p inhibitor abrogated these effects. These observations demonstrated that miR-126-3p could directly target PIK3R2 and then inhibit apoptosis and promote proliferation of porcine ovarian GCs.

In this study, we found that siRNA-PIK3R2 inhibited apoptosis (Figure 2B) and promoted proliferation (Figure 2C) of ovarian GCs. The result was in accordance with previous study where they found that knockdown PIK3R2 could inhibit the apoptosis and promote the proliferation of rheumatoid arthritis synovial fibro-blasts cell. PI3K is a phosphatidylinositol-like compound which acts as the second messenger in the growth signaling pathway. Furthermore, studies have shown that PI3K signaling pathway may be involved in differentiation and proliferation of ovarian GCs, the selection and recruitment of luminal follicles, and the biological processes of mature follicle ovulation [34-36]. In this study, we also confirmed that interference with PIK3R 2 could significantly decrease expression levels of key genes on the PI3K signaling pathway, such as IGF1R, INSR, PDK1, and AKT1. Both PDK1 and AKT1 were positive regulators of primordial follicle activation, which stimulate the activation of the PI3K signaling pathway [37]. Studies have reported that when PDK1 was knockout from oocytes of primordial follicles in mice, the majority of primordial follicles died directly from their dormant state around the onset of sexual maturity [38]. In AKT1-null mice, the number of growing antral follicles are reduced, while the number of degenerated oocytes are increased [39]. In IGF1-null mice, there are no mature large antral follicles produced and eventually resulting in infertile $[40,41]$. Compared with GCs of follicular cysts in cows, a recent study reported that INSR and PI3K were significantly higher expressed in GCs of control antral follicles. This indicated INSR and PI3K might involve in the regulation of steroidogenic enzymes expression [42].

In conclusion, we found miR-126-3p could directly target at PIK3R2 and inhibit apoptosis and promote proliferation of porcine ovarian GCs by down-regulating several key genes of the PI3K pathway, such as IGF1R, INSR, PDK1, and AKT1 (Figure 4). This observation will help further understanding of the molecular mechanism of miR-126-3p function and its own expression regulation in porcine ovarian GCs.

\section{CONFLICT OF INTEREST}

We certify that there is no conflict of interest with any financial organization regarding the material discussed in the manuscript.

\section{ACKNOWLEDGMENTS}

This work was supported by the special fund for science and technology innovation of Guangdong Province (2018B02020 3003), the National Natural Science Foundation of China (31902131), the National Natural Science Foundation of Guangdong Province (2019A1515010676), the Youth Innovation fund of Guangdong Education Department (2018 KQNCX019), and the earmarked fund for the China Agri- 
culture Research System (CARS-35).

\section{REFERENCES}

1. Pepling ME, Spradling AC. Mouse ovarian germ cell cysts undergo programmed breakdown to form primordial follicles. Dev Biol 2001;234:339-51. https://doi.org/10.1006/dbio.2001. 0269

2. McGee EA, Hsueh AJW. Initial and cyclic recruitment of ovarian follicles. Endocr Rev 2000;21:200-14. https://doi.org/ 10.1210/edrv.21.2.0394

3. Reddy P, Shen L, Ren C, et al. Activation of Akt (PKB) and suppression of FKHRL1 in mouse and rat oocytes by stem cell factor during follicular activation and development. Dev Biol 2005;281:160-70. https://doi.org/10.1016/j.ydbio.2005. 02.013

4. Fiedler SD, Carletti MZ, Hong XM, Christenson LK. Hormonal regulation of microRNA expression in periovulatory mouse mural granulosa cells. Biol Reprod 2008;79:1030-7. https:// doi.org/10.1095/biolreprod.108.069690

5. Eppig JJ. Oocyte control of ovarian follicular development and function in mammals. Reproduction 2001;122:829-38. https://doi.org/10.1530/rep.0.1220829

6. Shimizu T, Kosaka N, Murayama C, Tetsuka M, Miyamoto A. Apelin and APJ receptor expression in granulosa and theca cells during different stages of follicular development in the bovine ovary: Involvement of apoptosis and hormonal regulation. Anim Reprod Sci 2009;116:28-37. https://doi.org/10. 1016/j.anireprosci.2009.01.009

7. Zhang P, Wang J, Lang H, et al. Knockdown of CREB1 promotes apoptosis and decreases estradiol synthesis in mouse granulosa cells. Biomed Pharmacother 2018;105:1141-6. https://doi.org/10.1016/j.biopha.2018.06.101

8. Maneixa L, Antonsonb P, Humireb P, et al. Estrogen receptor $\beta$ exon 3-deleted mouse: The importance of non-ERE pathways in ER $\beta$ signaling. Proc Natl Acad Sci USA 2015;112: 5135-40. https://doi.org/10.1073/pnas.1504944112

9. Woodruff TK, Mayo KE. To beta or not to beta: estrogen receptors and ovarian function. Endocrinology 2005;146:32446. https://doi.org/10.1210/en.2005-0630

10. Rodriguez KF, Couse JF, Jayes FL, et al. Insufficient luteinizing hormone-induced intracellular signaling disrupts ovulation in preovulatory follicles lacking estrogen receptor- $\beta$. Endocrinology 2010;151:2826-34. https://doi.org/10.1210/en.2009-1446

11.Lei L, Jin S, Gonzalez G, Behringer RR, Woodruff TK. The regulatory role of Dicer in folliculogenesis in mice. Mol Cell Endocrinol 2010;315:63-73. https://doi.org/10.1016/j.mce. 2009.09.021

12.Hamm ML, Bhat GK, Thompson WE, Mann DR. Folliculogenesis is impaired and granulosa cell apoptosis is increased in leptin deficient mice. Biol Reprod 2004;71:66-72. https:// doi.org/10.1095/biolreprod.104.027292
13. Kim VN, Han J, Siomi MC. Biogenesis of small RNAs in animals. Nat Rev Mol Cell Biol 2009;10:126-39. https://doi.org/ $10.1038 / \mathrm{nrm} 2632$

14.Hong X, Luense LJ, McGinnis LK, Nothnick WB, Christenson LK. Dicerl is essential for female fertility and normal development of the female reproductive system. Endocrinology 2008;149:6207-12. https://doi.org/10.1210/en.2008-0294

15.Nagaraja AK, Andreu-Vieyra C, Franco HL, et al. Deletion of Dicer in somatic cells of the female reproductive tract causes sterility. Mol Endocrinol 2008;22:2336-52. https://doi. org/10.1210/me.2008-0142

16. Yao G, Yin M, Lian J, et al. MicroRNA-224 is involved in transforming growth factor-beta-mediated mouse granulosa cell proliferation and granulosa cell function by targeting Smad4. Mol Endocrinol 2010;24:540-51. https://doi.org/10.1210/me. 2009-0432

17.Lin F, Li R, Pan ZX, et al. miR-26b promotes granulosa cell apoptosis by targeting ATM during follicular atresia in porcine ovary. PLoS ONE 2012;7:e38640. https://doi.org/10.1371/ journal.pone.0038640

18. Bernstein C, Bernstein H, Payne CM, Garewal H. DNA repair/ pro-apoptotic dual-role proteins in five major DNA repair pathways: fail-safe protection against carcinogenesis. Mutat Res 2002;511:145-78. https://doi.org/10.1016/S1383-5742 (02)00009-1

19. Ma T, Jiang H, Gao Y, et al. Microarray analysis of differentially expressed microRNAs in non-regressed and regressed bovine corpus luteum tissue; microRNA-378 may suppress luteal cell apoptosis by targeting the interferon gamma receptor 1 gene. J Appl Genet 2011;52:481-6. https://doi.org/10.1007/ s13353-011-0055-Z

20. Mao Z, Fan L, Yu Q, et al. Abnormality of klotho signaling is involved in polycystic ovary syndrome. Reprod Sci 2018;25: 372-83. https://doi.org/10.1177/1933719117715129

21. Yuan X, Deng X, Zhou X, et al. MiR-126-3p promotes the cell proliferation and inhibits the cell apoptosis by targeting TSC1 in the porcine granulosa cells. In Vitro Cell Dev Biol Anim 2018;54:715-24. https://doi.org/10.1007/s11626-0180292-0

22. Qu Y, Wu J, Deng JX, et al. MicroRNA-126 affects rheumatoid arthritis synovial fibroblast proliferation and apoptosis by targeting PIK3R2 and regulating PI3K-AKT signal pathway. Oncotarget 2016;7:74217-26. https://doi.org/10.18632/onco target.12487

23. Wu XJ, Zhao ZF, Kang XJ, Wang HJ, Zhao J, Pu XM. MicroRNA126-3p suppresses cell proliferation by targeting PIK3R2 in Kaposi's sarcoma cells. Oncotarget 2016;7:36614-21. https:// doi.org/10.18632/oncotarget.9311

24. Sessa R, Seano G, di Blasio L, et al. The miR-126 regulates angiopoietin-1 signaling and vessel maturation by targeting p85beta. Biochim Biophys Acta 2012;1823:1925-35. https:// doi.org/10.1016/j.bbamcr.2012.07.011 
25. Gao J, Zhou XL, Kong RN, Ji LM, He LL, Zhao DB. microRNA -126 targeting PIK3R2 promotes rheumatoid arthritis synovial fibro-blasts proliferation and resistance to apoptosis by regulating PI3K/AKT pathway. Exp Mol Pathol 2016;100:192-8. https://doi.org/10.1016/j.yexmp.2015.12.015

26. Rodrigues Alves APN, Fernandes JC, Fenerich BA, et al. IGF1R/IRS1 targeting has cytotoxic activity and inhibits PI3K/ $\mathrm{AKT} / \mathrm{mTOR}$ and MAPK signaling in acute lymphoblastic leukemia cells. Cancer Lett 2019;456:59-68. https://doi.org/ 10.1016/j.canlet.2019.04.030

27. Bai X, Chen XH, Liu YH, et al. Effects of water extract and crude polysaccharides from Liriope spicata var. prolifera on InsR/IRS-1/PI3K pathway and glucose metabolism in mice. J Ethnopharmacol 2009;125:482-6. https://doi.org/10.1016/ j.jep.2009.07.007

28.Sambandam V, Frederick MJ, Shen L, et al. PDK1 mediates NOTCH1-mutated head and neck squamous carcinoma vulnerability to therapeutic PI3K/mTOR inhibition. Clin Cancer Res 2019;25:3329-40. https://doi.org/10.1158/10780432.CCR-18-3276

29. Wang B, Zhao CH, Sun G, et al. IL-17 induces the proliferation and migration of glioma cells through the activation of PI3K/ Akt1/NF-kappa B-p65. Cancer Lett 2019;447:93-104. https:// doi.org/10.1016/j.canlet.2019.01.008

30.Dai L, Xu J, Liu S, et al. Characterization of miR-126-3p and its target talin2 in the bovine corpus luteum during the oestrus cycle. Reprod Domest Anim 2014;49:913-9. https://doi.org/ 10.1111/rda. 12400

31.Cui W, Li Q, Feng L, Ding W. MiR-126-3p regulates progesterone receptors and involves development and lactation of mouse mammary gland. Mol Cell Biochem 2011;355:17-25. https://doi.org/10.1007/s11010-011-0834-1

32. Sirotkin AV, Laukova M, Ovcharenko D, Brenaut P, Mlynček M. Identification of microRNAs controlling human ovarian cell proliferation and apoptosis. J Cell Physiol 2010;223:49-56. https://doi.org/10.1002/jcp.21999

33. Sirotkin AV, Ovcharenko D, Grossmann R, Lauková M, Mlynček M. Identification of MicroRNAs controlling human ovarian cell steroidogenesis via a genome-scale screen. J Cell Physiol 2009;219:415-20. https://doi.org/10.1002/jcp.21689

34. Fan HY, Richards JS. Minireview: Physiological and pathological actions of RAS in the ovary. Mol Endocrinol 2010;24: 286-98. https://doi.org/10.1210/me.2009-0251

35.Zeleznik AJ, Saxena D, Little-Ihrig L. Protein kinase B is obligatory for follicle-stimulating hormone-induced granulosa cell differentiation. Endocrinology 2003;144:3985-94. https:// doi.org/10.1210/en.2003-0293

36. Gonzalez-Robayna IJ, Falender AE, Ochsner S, Firestone GL, Richards JS. Follicle-stimulating hormone (FSH) stimulates phosphorylation and activation of protein kinase $\mathrm{B}$ (PKB/ Akt) and serum and glucocorticoid-induced kinase (Sgk): evidence for a kinaseindependent signaling by FSH in granulosa cells. Mol Endocrinol 2000;14:1283-300. https://doi.org/10. 1210/mend.14.8.0500

37.Zheng W, Nagaraju G, Liu Z, Liu K. Functional roles of the phosphatidylinositol 3-kinases (PI3Ks) signaling in the mammalian ovary. Mol Cell Endocrinol 2012;356:24-30. https:// doi.org/10.1016/j.mce.2011.05.027

38. Reddy P, Adhikari D, Zheng W, et al. PDK1 signaling in oocytes controls reproductive aging and lifespan by manipulating the survival of primordial follicles. Hum Mol Genet 2009;18:281324. https://doi.org/10.1093/hmg/ddp217

39. Brown C, LaRocca J, Pietruska J, et al. Subfertility caused by altered follicular development and oocyte growth in female mice lacking PKBalpha/Akt1. Biol Reprod 2010;82:246-56. https://doi.org/10.1095/biolreprod.109.077925

40. Baker J, Hardy MP, Zhou J, et al. Effects of an Igf1 gene null mutation on mouse reproduction. Mol Endocrinol 1996;10: 903-18. https://doi.org/10.1210/mend.10.7.8813730

41. Bachelot A, Monget $P$, Imbert-Bolloré $P$, et al. Growth hormone is required for ovarian follicular growth. Endocrinology 2002; 143:4104-12. https://doi.org/10.1210/en.2002-220087

42. Gareis NC, Huber E, Hein GJ, et al. Impaired insulin signaling pathways affect ovarian steroidogenesis in cows with COD. Anim Reprod Sci 2018;192:298-312. https://doi.org/10.1016/ j.anireprosci.2018.03.031 